Ann. Geophys. Discuss., https://doi.org/10.5194/angeo-2019-64

Manuscript under review for journal Ann. Geophys.

Discussion started: 26 April 2019

(c) Author(s) 2019. CC BY 4.0 License.

\title{
Characteristics of ionospheric irregularities near the northern equatorial anomaly crest
}

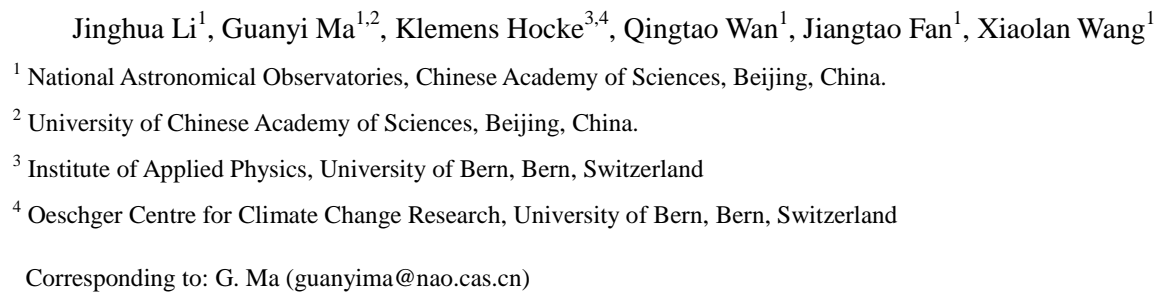

Abstract. This paper detects the ionospheric irregularities with rate of total electron content (TEC) change index, ROTI from GPS observation at Taoyuan $\left(24.95^{\circ} \mathrm{N}, 121.16^{\circ} \mathrm{E}\right)$ for the solar medium and minimum years of 2003 and 2008 in the declining phase of cycle 23, the solar maximum of 2014 in solar cycle 24. Local occurrence rate (LOR) is proposed to clarify the characteristics of the irregularities together with monthly occurrence rate (MOR) and ROTI maximum for 3 latitude belts, $20-23^{\circ} \mathrm{N}, 23-26^{\circ} \mathrm{N}, 26-29^{\circ} \mathrm{N}$, around the equatorial anomaly crest. MOR in May/June is larger than those in equinoxes in 2008 and 2003, which is different from that of equatorial plasma bubbles. In 2014 although MOR maximum is observed in equinoxes, the MOR in May and June is much larger than that in September. Moreover, MORs in May to August at higher latitude belt $26-29^{\circ} \mathrm{N}$ are larger than those in lower latitude belts and smaller in the equinoxes. The latitudinal dependence of the LORs tends to be similar to that of MORs. Seasonal variations of LORs have a similar trend for different solar activities. Maximum LORs are observed in Feb/Mar and Sep/Oct, and moderate around June, which resemble those of plasma bubbles in seasonal variations, except for latitude belt $26-29^{\circ} \mathrm{N}$ where maximum LORs are seen in May-Jul. The seasonal variation of ROTI maximum conforms to that of the LOR. The results suggest that irregularities near the crest in May to August are mainly originated from nonequatorial process, which is more frequently happened but weaker than plasma bubble in both spatiotemporal scale and strength.

\section{Introduction}

The ionospheric irregularities are spatially irregular variation of electron density or fluctuation of total electron content (TEC) with scale lengths from a few meters to several tens of kilometers. When radiowaves propagate through the irregularities, variations in signal strength happen due to refractive effect. This phenomenon is referred to as ionospheric scintillation, which 
Ann. Geophys. Discuss., https://doi.org/10.5194/angeo-2019-64

Manuscript under review for journal Ann. Geophys.

Discussion started: 26 April 2019

(c) Author(s) 2019. CC BY 4.0 License.

degrades both the performance of satellite communication and the precision of satellite navigation (Basu \& Basu, 1981; Maruyama, 2002). Severe scintillation can lead satellite service interruption due to loss of lock in receivers. Moreover, TEC fluctuations in ionospheric irregularities are a major error to radio interferometers, differential Global Positioning System (GPS) and synthetic aperture radar (SAR) (Erickson et al., 2001; Zabotin and Wright, 2004; Afraimovich and Yasukevich, 2008; Lee et al., 2011, Zheng et al., 2008).

Most intense ionospheric irregularities are those occurred in low-latitude regions near the magnetic equator. They were first recorded as the spreading of the traces of $\mathrm{F}$ layer echoes on ionograms with ionosonde. It has been since called equatorial spread $\mathrm{F}$ as well (Booker and Wells, 1938). Using the data from incoherent scattering radar, a plumelike irregular structure was found in a range-time-intensity diagram which provides the dynamic process of the irregularity evolution. It was then proposed that the irregularities are produced as low-density 'plasma bubbles' at the bottomside of the ionosphere and rose to an altitude of $1000 \mathrm{~km}$ (Woodman and La Hoz, 1976). The equatorial plasma bubbles (EPB) were directly confirmed by AE-C satellite in-situ measurements as regions of abrupt drop-out of electron density by two orders of magnitude with sizes of several tens of kilometers (McClure et al., 1977). Furthermore the optical imaging techniques probed two-dimensional structure of the plasma bubble (Weber et al., 1978; Mendilo and Baumgardner, 1982; Balan et al., 2018). It has been generally understood that plasma bubbles are generated at the bottom of the equatorial ionosphere by the generalized nonlinear Rayleigh-Taylor instability. While moving upward from the lower ionosphere into the higher density ionosphere, they extend along the magnetic flux tube to higher latitudes and often reach to the equatorial anomaly crest (Kelley and McClure, 1981; Ossakow, 1981).

The ionospheric irregularities have been studied with radar, satellite, airglow imager observations and simulations (Balan et al., 2018). Different observational techniques have their own advantage to reveal different aspects of the characteristics of the ionospheric irregularity. Since the civilian use of Global Navigation Satellite System (GNSS) the observation with ground-based dual-frequency GNSS receivers has become an important mean for ionospheric studies. While the fluctuation of phase and amplitude of GNSS signal, and hence scintillation, occur due to ionospheric irregularity, TEC also fluctuates. The rate of change of the TEC, termed ROT, and further, a rate of TEC index (ROTI) have been proposed as an indicator of presence of 
Ann. Geophys. Discuss., https://doi.org/10.5194/angeo-2019-64

Manuscript under review for journal Ann. Geophys.

Discussion started: 26 April 2019

(c) Author(s) 2019. CC BY 4.0 License.

ionospheric irregularity and a surrogate of TEC fluctuation (Aaron et al., 1996; Pi et al. 1997; Basu et al., 1999). ROTI was used to investigate extensively the ionospheric equatorial bubbles or irregularities at different time and over different regions for the last 20 years. Statistical and case studies were successful in describing the occurrence features of the irregularity with local time, seasonal variations, solar cycle and its geographical dependence. They confirm already existing observational characteristics of the irregularity and give new insights into its global distribution scenarios (Otsuka et al., 2006, Nishoka et al., 2008; Sripathi et al., 2018). Nevertheless, systematic research of the ionospheric irregularity with ROTI is lacking at specific area for varying solar activity. It is difficult to coordinate the available results since different research groups use different definition in their studies (Mendillo et al., 2000; Otsuka et al., 2006; Nishioka et al., 2008). Moreover, the strength of the irregularity or TEC fluctuation has not yet been addressed quantitatively.

This paper aims to study statistical characteristics of plasma bubble associated irregularities near the equatorial anomaly crest with continuous GPS observations for the solar minimum of 2008 , medium of 2003 in the declining phase of cycle 23, and the solar maximum of 2014 in solar cycle 24. Section 2 describes observation and data analysis method. Section 3 gives the results. Section 4 deals with the discussion. Finally, conclusions are drawn in section 5.

\section{Observation and data analysis}

\subsection{Observation}

The GPS system composes of 24 to 32 satellites (on 6 planes) orbiting the Earth at an inclination of $55^{\circ}$ and at a height of $20200 \mathrm{~km}$. Each satellite broadcasts information on two frequency carrier signals, which are $1.57542 \mathrm{GHz}$ (referred to as $f_{1}$ ) and $1.2276 \mathrm{GHz}$ (referred to as $f_{2}$ ), respectively. Containing dozen or more parallel channels, a dual-frequency GPS receiver is capable of receiving GPS signals from $\sim 10$ GPS satellites simultaneously. The observation is from a dual-frequency GPS receiver with 30-s sampling rate taken by the International GNSS Service (IGS) at Taoyuan $\left(24.95^{\circ} \mathrm{N}, 121.16^{\circ} \mathrm{E}\right)$, Taiwan, which is the ionospheric equatorial anomaly crest with frequent occurrence of irregularities. The data are from the solar medium of 
Ann. Geophys. Discuss., https://doi.org/10.5194/angeo-2019-64

Manuscript under review for journal Ann. Geophys.

Discussion started: 26 April 2019

(c) Author(s) 2019. CC BY 4.0 License.

solar cycle 24 .

Figure 1 depicts a map of the IPP tracks of GPS satellites at $400 \mathrm{~km}$ observed by Taoyuan GPS receiver, with an elevation cutoff of 30 degrees, during 18:00 06:00 local time on 20th March 2003. The starting positions of the traces are marked with dots. The directions of the traces can be southward, northward, eastward, northeastward, or southeastward, except any westward orientation. The coverage of these traces is mainly within $20-29^{\circ} \mathrm{N}$ in latitude and $116-126^{\circ} \mathrm{E}$ in longitude. Due to the arrangement of the satellites and their periodic orbits, the coverage of the IPP traces is almost the same every day.

\subsection{ROTI calculation}

Under the irregularity "frozen-in" assumption, the characteristics of the ionospheric irregularities do not change within a short time when the ray path from a GPS satellite to a receiver traverses them. The TEC along the ray path would vary correspondingly due to the TEC fluctuation. By taking the difference between the slant TECs at two successive times, a rate of change of the TEC was defined as $R O T_{i}=\left(N_{\pi_{i}}-N_{\pi-1}\right) /\left(t_{i}-t_{i-1}\right)$ where $N_{\pi i}$ is the slant TEC for the ionosphere, ROT is a time changing parameter. Further, ROTI, the standard deviation of ROT, is calculated to quantify the TEC fluctuation with time and space, $R O T I=\sqrt{\left\langle R O T_{i}{ }^{2}\right\rangle-\left\langle R O T_{i}\right\rangle^{2}}$, where (4) is the mean operator. With 30 -s time series of GPS data, ROTI is generally calculated on a 5-min time window (Pi et al., 1997).

\subsection{Detection of irregularity traverse event}

The ionospheric irregularity is surveyed by a criterion as follows. The GPS observation with satellite elevation larger than 30 degrees is used in order to mitigate the effect of the multipath. ROTI is calculated on a 5-min time window with 11 successive data. An irregularity encounter is happened if ROTI is larger than a threshold. The threshold for the irregularity is the sum of the 
Ann. Geophys. Discuss., https://doi.org/10.5194/angeo-2019-64

Manuscript under review for journal Ann. Geophys.

Discussion started: 26 April 2019

(c) Author(s) 2019. CC BY 4.0 License.

traverse the ionospheric irregularities, and an irregularity traverse event is identified. Another irregularity traverse event will be counted if it is encountered more than $1 \mathrm{~h}$ later than the first or preceding event. Although the threshold can be a little different day by day, it is in the range of 0.11-0.20 TECU/min for all the irregularity events in this study.

\subsection{Occurrence rates definitions}

Two kinds of occurrence rates are defined and studied. Monthly occurrence rate (MOR) is defined by the ratio of days with irregularity traverse observed to the days the observation is made. Higher monthly occurrence rate implies the irregularity happens frequently in daily scale. Local occurrence rate (LOR) is defined by the ratio of numbers of the irregularity encounters of all irregularity traverses to the numbers of all ROTI calculated between 17:00-7:00 LT. To compare with monthly occurrence rate, the local occurrence rate is counted based on one month. Higher local occurrence rate means the irregularity tends to exist with larger spatial and temporal scales.

\section{Results and discussion}

\subsection{Start time of irregularity traverse}

For the three years studied, 306 irregularity traverse events were identified. The distribution of the start time observed for the irregularity traverse is shown in Fig. 2. It is noteworthy that since the traces of the IPP can be any direction except westward orientation, the first time an irregularity encounter observed is not necessarily the onset time of the irregularity. Moreover, the irregularities observed in the same traverse event are not necessarily from the same source. From Fig. 2, most of the irregularity traverses are first observed after sunset and before midnight.

In 2003 there were 98 irregularity traverses, of which 71 (72\%) were first seen at the time within 18:00-24:00 LT. In 2008, 33 out of 40 (82\%) arose within 18:00-24:00 LT. It is 135 out of 168 events $(80 \%)$ in 2014. The first observed times of irregularity traverses in 2014 have a prominent peak of 38 corresponding to 19:00-20:00 LT bin. The 38 irregularity traverses are mostly from Feb. and Mar. While there were only 7 (18\%) irregularity traverses happened after midnight in 2008, there were $25(26 \%)$ and $32(19 \%)$ irregularity traverses observed later than midnight in 2003 and 2014, respectively. 
Ann. Geophys. Discuss., https://doi.org/10.5194/angeo-2019-64

Manuscript under review for journal Ann. Geophys.

Discussion started: 26 April 2019

(c) Author(s) 2019. CC BY 4.0 License.

\subsection{Variation of Occurrence rates}

Figure 3 shows the monthly occurrence rates (higher row) and the local occurrence rates (lower row) for solar minimum (2008), medium (2003) and maximum (2014). By dividing the area into 3 latitude belts, $20-23^{\circ} \mathrm{N}, 23-26^{\circ} \mathrm{N}, 26-29^{\circ} \mathrm{N}$, latitudinal dependence of the irregularity occurrence can be also studied. The seasonal variation of MOR has a clear dependence on solar activity. In 2008, the MOR has peaks of $\sim 17 \%-25 \%$ and increases with latitude belt in May. There is no irregularity observed in March and November for all the area. The MOR peaks in May/Jun and is generally larger in May to July than other months in 2003. The value of the peaks ranges from $35 \%$ to $\sim 45 \%$. Moderate maximum MORs can be seen in Feb/Oct, except for that of latitude belt $26-29$. In 2014 , the peaks range from $\sim 45 \%$ to $72 \%$ in March; from $\sim 55 \%$ to $\sim 63 \%$ in June; and from $\sim 10 \%$ to $\sim 35 \%$ in September. It is noteworthy that the MOR for latitude belt $26-29^{\circ} \mathrm{N}$ has the largest maximum in June and no maximum in autumn. Concerning latitude dependence, the MOR increases with latitude in May/Jun. It is generally the largest in May to August for latitude belt $26-29^{\circ} \mathrm{N}$. It deceases with latitude in equinoxes and winter months, and this is especially true in 2014.

It is obvious that the LOR behaves very different from the MOR. LOR is much smaller than MOR. While the largest MOR can be $72 \%$ for latitude belt $20-23^{\circ} \mathrm{N}$ in March 2014 , the largest LOR at the same time and place is only $\sim 15 \%$. The LOR peaks dominantly in Feb/Mar and Sep/Oct except for the latitude belt $26-29^{\circ} \mathrm{N}$ where the LOR shows larger maximum in June of both 2003 and 2014, and July of 2008. The LOR t is generally larger in Feb/Mar than Sep/Oct, although the result is reversed for the latitude belt $20-23^{\circ} \mathrm{N}$ in 2003 . In 2008 , the LOR peaks in is one month later than autumn equinox. Though the LORs tend to be larger in higher solar activity year, the differences of maximum LORs are small for different solar activity years. This suggests whether the irregularity at Taoyuan area happens frequently or not, their spatial and temporal scales are rather independent of solar activity. Similar to MOR's latitude dependence, the 
Ann. Geophys. Discuss., https://doi.org/10.5194/angeo-2019-64

Manuscript under review for journal Ann. Geophys.

Discussion started: 26 April 2019

(c) Author(s) 2019. CC BY 4.0 License.

\subsection{Variation of ROTI Maximum}

ROTI generally starts from a low value, increases and reaches to a maximum, and then deceases with the evolution of irregularities. ROTI maximum for an irregularity traverse event can be an indicator of irregularity strength. Figure 4 displays the variation of ROTI maximum for different latitude belts in the three different solar activity years. In solar minimum year of 2008 , the ROTI maximum is very low and peaks in February with values of $\sim 0.65-1.58 \mathrm{TECU} / \mathrm{min}$ for different latitude belts. It is around or below $0.50 \mathrm{TECU} / \mathrm{min}$ for other months. The differences of ROTI maximums among different latitude belts are generally small. In solar medium year of 2003, and 1.84-3.42 TECU/min, respectively. ROTI maximum for latitude belt $20-23^{\circ} \mathrm{N}$ also has peaks at May and August. The ROTI maximum increases with latitude in March, it can also decrease with latitude in April-July, October and November. In solar maximum year of 2014, ROTI maximum has peaks of 4.61-8.29 TECU/min in Feb/Mar and of 2.25-4.86 TECU/min in Sep. The ROTI maximum decreases with latitude in Feb/Mar. The largest ROTI maximum happens in latitude belt $23-26^{\circ} \mathrm{N}$ in September. ROTI maximum increases with latitude in June and the peak value of $2.28 \mathrm{TECU} / \mathrm{min}$ for latitude belt $26-29^{\circ} \mathrm{N}$ is comparatively large. The ROTI maximum for latitude belt $20-23^{\circ} \mathrm{N}$ has a moderate peak of $4.55 \mathrm{TECU} / \mathrm{min}$ in November. There are also peaks in September, although the values of ROTI maximum are around $3.84 \mathrm{TECU} / \mathrm{min}$, which is smaller than that in May. ROTI maximum in $26-29^{\circ} \mathrm{N}$ latitude belt is generally smaller than those in other latitude belts for the whole year except in June. Overall, the seasonal variation of ROTI maximum conforms to that of the LOR. ROTI maximum tends to be larger for higher solar activity.

\subsection{ROTI maximum with solar activity}

A scatter plot was attempted to examine in detail the relation of ROTI maximums for irregularity traverses with solar activity, as shown in Fig. 5. Here used is the radio flux at $10.7 \mathrm{~cm}$ (F10.7) as an indicator of the solar activity. The symbols of cross, circle and dot represent the year of 2003, 2008 and 2014, respectively. The colors in red, magenta, green and blue distinguish spring, summer, autumn and winter. In solar minimum of 2008, F10.7 is smaller than $80 \mathrm{sfu}$. 
Ann. Geophys. Discuss., https://doi.org/10.5194/angeo-2019-64

Manuscript under review for journal Ann. Geophys.

Discussion started: 26 April 2019

(c) Author(s) 2019. CC BY 4.0 License.

ROTI was generally around $0.50 \mathrm{TECU} / \mathrm{min}$. The largest ROTI is $1.64 \mathrm{TECU} / \mathrm{min}$ in winter. In solar medium and maximum years of 2003 and 2014, F10.7 is between 84-172 sfu and 86-208 sfu, respectively. ROTI is from 0.24 to $7.62 \mathrm{TECU} / \mathrm{min}$ in 2003 . The largest value of ROTI is 8.82 TECU/min in winter of 2014, corresponding to $175 \mathrm{sfu}$. ROTI can be small (less than 1 TECU/min) however large F10.7 is, especially in summer. For F10.7 larger than 200 sfu in summer, ROTI maximum is very small. ROTI can increase with F10.7 in all seasons of both 2003 and 2014. ROTI maximum tends to increase with F10.7 for those larger than 140 sfu, except for summer in 2003 and 2014. As a whole the scatter was confined in a funnel. A large ROTI maximum has a tendency to be related with a large F10.7, but the reverse is not always true.

\section{Discussions}

Low latitude irregularities are generally thought to be related with plasma bubbles generated at the magnetic equator. The occurrence of plasma bubble in Asian region is known to be maximum in equinoxes when sunsets in the conjugate E-regions are simultaneous (Tsunoda, 1985). Nishioka et al. (2008) revealed higher monthly occurrence rate of plasma bubble around spring equinox (summer solstice) than autumn equinox (winter solstice) from 2000 to 2006 with ground-based GPS networks. Buhari et al. (2017) studied the occurrence rate of plasma bubble with Malaysia Real-Time Kinematics Network (MyRTKnet) from 2008 to 2013. They found that the occurrence day of EPB remains active during equinoctial months in low solar activity years.

In a morphology study of equatorial plasma bubbles during low and high solar activity years in latitudes $13-17^{\circ} \mathrm{N}$ over Indian sector, Kumar (2017) found maximum EPB occurrences during the equinoctial months and minimum during the December solstice throughout 2007-2012 except during the solar minimum years in 2007-2009. During 2007-2009, the maximum EPB occurrences were observed in June solstice. Observations of equatorial and low latitude irregularities with a meridional chain of ionosondes and GPS receivers in 2015 showed that maximum EPB occurrences during the equinoctial months and minimum during the December solstice throughout 2007-2012 except during the solar minimum years in 2007-2009. During 2007-2009, the maximum EPB occurrences were observed in June solstice (Kumar, 2017). As for the equatorial anomaly crest, Lee et al. (2009) investigated the occurrence probabilities of 
Ann. Geophys. Discuss., https://doi.org/10.5194/angeo-2019-64

Manuscript under review for journal Ann. Geophys.

Discussion started: 26 April 2019

(c) Author(s) 2019. CC BY 4.0 License.

irregularity in solar maximum of 2000 with spread F, GPS phase fluctuations, and plasma bubbles concurrently for the first time. The data used are mainly from the ionosonde and GPS receiver at Chungli $\left(24.9^{\circ} \mathrm{N}, 121.2^{\circ} \mathrm{E}\right)$ which is very close to Taoyuan, ROCSAT-1, and three GPS receivers located at positions $\left(14.0-14.6^{\circ} \mathrm{N}, 121.0-121.1^{\circ} \mathrm{E}\right)$ in Manila. To detect the irregularity traverse event with GPS observation, they used an index Fp which is also based on rate of change of the TEC, ROT, but takes the average median of ROT for all the satellites (refer to Mendillo et al., 2000 for details). They found that the seasonal variations in GPS phase fluctuations at the crest and the dip equator had similar trends in solar maximum of year 2000. They also showed the range spread F (RSF) had similar occurrence probability to that of GPS phase fluctuations while the frequency spread F (FSF) peaked in June as the spread $\mathrm{F}$ at mid latitude.

In Fig. 2, the MOR in solar maximum year of 2014 generally shows maximum values in March, June, September, and November, respectively. The maximum value in March (June) is much larger than that in September (November) for latitudes $20-26^{\circ} \mathrm{N}$. This is in agreement with the results from GPS observations both at equatorial region and crest in solar maximum and high activity years (Nishioka et al., 2008; Kumar, 2017; Lee et al., 2009). However, the MOR for latitude $26-29^{\circ} \mathrm{N}$ is larger in May-June than March in 2014, which is different from that of plasma bubble. It is also noteworthy that the maximum values decrease with latitude, except that in May to August which mainly increase with latitude. With ROTI from GPS observations in solar maximum year of 2000 in Japan, Otsuka et al. (2006) showed similar results that the occurrence rate peaks at equinoxes in $25^{\circ} \mathrm{N}$, but peaks at summer in $29^{\circ} \mathrm{N}$. In both solar medium 2003 and minimum 2008, the MOR just shows maximum values in May and keeps larger values until August. MOR increases with latitude, or MOR is the largest at $26-29^{\circ} \mathrm{N}$ latitude belt implies that the irregularity is encountered more frequently at higher latitude. Kumar (2017) also reported maximum MOR in June observed with GPS receivers in latitudes $13-17^{\circ} \mathrm{N}$ over India for 2007-2009. However, Buhari et al. (2017) found that the MOR of EPB over Malaysia (near magnetic equator) was still active during equinoctial months in low solar activity years. So the MOR of irregularity observed with Taoyuan GPS receivers is completely different from that of 
For the LOR, similar scenario of plasma bubble can be seen regardless of solar activity level. As shown in Fig. 2, the LOR in solar maximum year of 2014 generally decreases with latitude, asymmetries are also there. In 2003 the LOR is more alike that of plasma bubble, although it has larger maximum value in October than March for latitude $20-23^{\circ} \mathrm{N}$. The two maximum and moderate peaks in 2008 are in February and November, respectively. They also decrease with latitude. However, the latitudinal feature of LOR in May to July is not the same as in other months.

The largest LOR is always in $26-29^{\circ} \mathrm{N}$ latitude belt for this period.

The physics behind the two occurrence rates behaviors can be explained with a schematic diagram of the earth's magnetic field lines at the meridian plane in Taoyuan as shown in Fig. 6. The intersections of the two solid bold magnetic field lines with the ionosphere at $400 \mathrm{~km}$ corresponds to latitudes of $20^{\circ} \mathrm{N}$ and $29^{\circ} \mathrm{N}$, respectively. If the plasma bubble reaches specific latitude, the irregularity occurrence rate should be the same from the latitude to the magnetic equator. Due to the day to day variability, the plasma bubble occurrence rate should decrease with latitude. For both monthly and local occurrence rates decreasing with latitude, it implies that the irregularities are related with magnetic equator originated plasma bubbles. This can apply to most of the irregularities observed in equinoxes (Feb/Mar and Sep/Oct) and winter solstices months for all the three different solar activity years. However, the latitudinal variation of MOR in May to August cannot be attributed to plasma bubble. Since the MOR increase with latitude, it can be that the irregularities originated from nonequatorial process, for example, TID coming from mid latitude, as shown in Fig. 6. On the other hand, the latitudinal variation of LOR in May to August is not quite the same as that of MOR. LOR in the lowest latitude belt $20-23^{\circ} \mathrm{N}$ is not always the smallest. LOR in latitude belt $26-29^{\circ} \mathrm{N}$ is always the largest in May to July. It can be concluded that there can be overlaps of irregularities originated from both plasma bubble and nonequatorial processes. The nonequatorial processes are more frequently happened but weaker in spatiotemporal scale than plasma bubbles in May to August. The irregularities in $26-29^{\circ} \mathrm{N}$ in May to July are mainly from nonequatorial processes. with a meridional chain of ionosondes and GPS receivers in 2015, which showed that the postmidnight spread F during summer was weaker in strength and shorter in duration than 
Ann. Geophys. Discuss., https://doi.org/10.5194/angeo-2019-64

Manuscript under review for journal Ann. Geophys.

Discussion started: 26 April 2019

(c) Author(s) 2019. CC BY 4.0 License.

equatorial spread $\mathrm{F}$ mostly occurred in equinoxes and winter. Further the postmidnight spread $\mathrm{F}$ during summer is found to be stronger and earlier at low latitudes followed by their occurrence at the equator (Sripathi et al., 2018).

Figure 4 shows the variation of the irregularity's strength generally complies with its LOR in Taoyuan, although a one-to-one correspondence does not exist coming to those of latitude belt. In 2008, the ROTI maximum peaks and deceases with latitude in February. It is generally weak for the whole year, implying the contribution from plasma bubble is less than that from nonequatorial processes. In solar medium and maximum the irregularity associated with plasma bubble can be very strong not only in March, but also in Feb and April. The ROTI maximum in May to August in 2003 is the smallest in latitude belt $26-29^{\circ} \mathrm{N}$, implying that the irregularity originated in nonequatorial processes is much weaker than plasma bubble. In 2014, more contribution from nonequatorial processes can be only seen in June. So, the nonequatorial origin irregularities are weaker (stronger) in strength than those related with plasma bubbles in solar medium and maximum (minimum) years. It is known that Perkins instability is responsible for the mid latitude irregularities (Perkins, 1973; Yokoyama et al., 2009). Otsuka et al. (2006) observed that the frequent occurrence of the irregularity in mid latitude in the summer night was usually accompanied by medium-scale traveling ionospheric disturbances (MSTID). Meridional observations from mid latitude to magnetic equator are needed to confirm and clarify the nonequatorial origin of the irregularities observed near equatorial anomaly crest.

The scatter plot of ROTI maximum versus F10.7 is rather dispersed, as shown in Fig. 5. However, confined in a funnel, a large ROTI maximum has a tendency to be related with a large F10.7, but the reverse is not always true. Higher solar activity is a necessary condition for the production of stronger plasma bubble related irregularities.

\section{Conclusion}

Making use of continuous GPS observations at Taoyuan $\left(24.95^{\circ} \mathrm{N}, 121.16^{\circ} \mathrm{E}\right)$, characteristics of plasma irregularities near the ionospheric anomaly crest are studied for the solar minimum of 2008, medium of 2003 in the declining phase of cycle 23, and the solar maximum of 2014 in solar cycle 24. The irregularity is surveyed and detected with ROTI. Most of the irregularities are first 
Ann. Geophys. Discuss., https://doi.org/10.5194/angeo-2019-64

Manuscript under review for journal Ann. Geophys.

Discussion started: 26 April 2019

(c) Author(s) 2019. CC BY 4.0 License.

observed after sunset and before midnight. Local occurrence rate (LOR) is proposed to clarify the sources of the irregularities together with Monthly occurrence rate (MOR) and ROTI maximum for 3 latitude belts, $20-23^{\circ} \mathrm{N}, 23-26^{\circ} \mathrm{N}, 26-29^{\circ} \mathrm{N}$, within $116-126^{\circ} \mathrm{E}$ longitude range. In 2014 , while maximum MORs are observed in equinoxes, which is similar to that of plasma bubbles, the MORs in May and June are much larger than those in September. In 2003 MORs are prominent in May-June, weaker maximums can be seen in February and October except for latitude belt $26-29^{\circ}$ N. In 2008 maximum MORs are only dominant in May. Moreover, MORs tend to be larger in higher latitude belts in May to August, while they tend to be smaller in higher latitude belts in other months. However, seasonal variations of LORs have a similar trend for different solar activities. Maximum LORs are observed in Feb/Mar and Sep/Oct, and moderate around June, which resembles those of plasma bubbles in seasonal variations, except for latitude belt $26-29^{\circ} \mathrm{N}$ where maximum LORs are seen in May-Jul. The latitudinal dependence of the LORs is not quite the same as that of MORs. The different behavior of MORs and LORs indicates that the irregularities in May to August are mainly originated from nonequatorial process, which is more frequently happened but weaker than plasma bubble in both spatial and temporal scales. The seasonal variation of ROTI maximum conforms to that of the LOR. ROTI maximum tends to be larger for higher solar activity. Nonequatorial origin irregularities are weaker (stronger) in strength than those related with plasma bubbles in solar medium and maximum (minimum) years. Higher solar activity is a necessary condition for the production of stronger plasma bubble related irregularities.

\section{Acknowledgement}

This research has been carried out under the support of the National Natural Science Foundation of China (NSFC No. 11873064) and National key Research Program of China "Collaborative Precision Positioning Project" (No.2016YFB0501900). IGS is acknowledged for free use of GPS 
Ann. Geophys. Discuss., https://doi.org/10.5194/angeo-2019-64

Manuscript under review for journal Ann. Geophys.

Discussion started: 26 April 2019

(c) Author(s) 2019. CC BY 4.0 License.

\section{References}

Aarons, J.: Global morphology of ionospheric scintillations, Proceedings of the IEEE, 70, 360-378, 1975.

Aarons, J., Mendillo, M., and Yantosca, R.: GPS phase fluctuations in the equatorial region during the MISETA 1994 campaign, Journal of Geophysical Research: Space Physics, 101, 26851-26862, 1996.

Abdu, M.A., Alam Kherani, E., Batista, I.S., de Paula, E.R., Fritts, D.C. and Sobral, J.H.A.: Gravity wave initiation of equatorial spread F/plasma bubble irregularities based on observational data from the SpreadFEx campaign, Annales Geophysicae, 27, 2607-2622, doi:10.5194/angeo-27-2607-2009, 2009.

Afraimovich, E.L. and Yasukevich, Yu.V.: Using GPSGLONASSGALILEO data and IRI modeling for ionospheric calibration of radio telescopes and radio interferometers, Journal of Atmospheric and Solar-Terrestrial Physics, 70, 1949-1962, 2008

Balan, N., Liu, L. B., and Le, H. J.: A brief review of equatorial ionization anomaly and ionospheric irregularities, Earth and Planetary Physics, 2, 257-275, 2018.

Basu, S. and Basu, S.: Equatorial scintillations-a review, Journal of Atmospheric and Terrestrial Physics, 43, 473-489, 1981.

Basu, S., Groves, K.M., Quinn, J.M., and Doherty, P.: A comparison of TEC fluctuations and scintillations at Ascension Island, Journal of Atmospheric and Solar-Terrestrial Physics, 61, 1219-1226, 1999.

Booker, H. G., and Wells, H. W.: Scattering of radio waves by the f-region of the ionosphere, Journal of Geophysical Research, 43, 249-256, 1938.

Buhari, S. M., Abdullah, M., Yokoyama, T., Otsuka, Y., Nishioka, M., Hasbi, A. M., Bahari, S. A., and Tsugawa, T., Climatology of successive equatorial plasma bubbles observed by GPS ROTI over Malaysia, Journal of Geophysical Research: Space Physics, 122, doi:10.1002/2016JA023202, 2017

Erickson, W. C., Perley, R. A., Flatters, C., and Kassim, N. E.: Ionospheric corrections for VLA observations using local GPS data, Astronomy and Astrophysics, 366, 1071-1080, doi:10.1051/0004-6361:20000359, 2001. 
Ann. Geophys. Discuss., https://doi.org/10.5194/angeo-2019-64

Manuscript under review for journal Ann. Geophys.

Discussion started: 26 April 2019

(c) Author(s) 2019. CC BY 4.0 License.

Kelley, M. C., and Mcclure, J. P.: Equatorial spread-F: A review of recent experimental results, Journal of Atmospheric and Terrestrial Physics, 43, 5, 427-435, 1981.

Kumar, S.: Morphology of equatorial plasma bubbles during low and high solar activity years over Indian sector, Astrophysics and Space Science, 362, 5, 93, 2017.

Lee, C. C., Chu, F. D., Chen, W. S., Liu, J. Y., Su, S.-Y., Liou Y. A., and Yu, S. B.: Spread F, GPS phase fluctuations, and plasma bubbles near the crest of equatorial ionization anomaly during solar maximum, Journal of Geophysical Research: Space Physics, 114, A08302, doi:10.1029/2009JA014195, 2009.

Lee, J., Amt, T. T., Jung, S., and Pullen, S.: Enhancements of Long Term Ionospheric Anomaly Monitoring for the Ground-Based Augmentation System, Proceedings of the 2011 International Technical Meeting of The Institute of Navigation, 930 San Diego, CA, January 24-26, 930-941, 2011.

Maruyama, T.: Ionospheric irregularities, Journal of the National Institute of Information and Communications Technology, 49, 163-179, 2002.

McClure, J. P., Hanson, W. B., and Hoffman, J. H.: Plasma bubbles and irregularities in the equatorial ionosphere, Journal of Geophysical Research: Space Physics, 82, 2650-2656, 1977.

Mendillo, M., and Baumgardner, J.: Airglow characteristics of equatorial plasma depletions, Journal of Geophysical Research: Space Physics, 87, 7641-7652, 1982.

Mendillo, M., Lin, B., and Aarons, J.: The application of gps observations to equatorial aeronomy, Radio Science, 35, 3, 885-904, 2000.

Nishioka, M., Saito, A., and Tsugawa, T.: Occurrence characteristics of plasma bubble derived from global groundbased GPS receiver networks, Journal of Geophysical Research: Space Physics, 113, A05301, doi:10.1029/2007JA012605, 2008.

Ossakow, S. L.: Spread-F theories-A review, Journal of Atmospheric and Solar-Terrestrial Physics, 43, 437-443, 1981.

Otsuka, Y., Aramaki, T., Ogawa, T., and Saito, A.: A statistical study of ionospheric irregularities observed with a GPS network in Japan, Corotating Solar Wind Streams and recurrent Geomagnetic Activity, Geophysical Monograph Series, AGU, Washington, D. C., 2006.

Perkins, F.: Spread F and ionospheric currents, Journal of Geophysical Research: Space Physics, 
Ann. Geophys. Discuss., https://doi.org/10.5194/angeo-2019-64

Manuscript under review for journal Ann. Geophys.

Discussion started: 26 April 2019

(c) Author(s) 2019. CC BY 4.0 License.

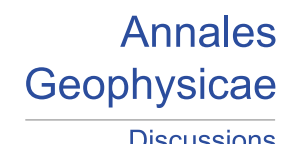

Discussions

(c) (i)

78, 218-226, 1973.

Pi X., Mannucci, A. J., Lindqwister, U. J., and Ho, C. M.: Monitoring of global ionospheric irregularities using the worldwide GPS network, Geophysical Research Letters, 24, 2283-2486, 1997.

Sripathi, S., Sreekumar, S. and Banola, S.: Characteristics of Equatorial and Low-Latitude Plasma Irregularities as Investigated Using a Meridional Chain of Radio Experiments Over India, Journal of Geophysical Research: Space Physics, 123, 9, DOI: 10.1029/2017JA024980, 2018.

Weber, E. J., Buchau, J., Eather, R. H., and Mende, S. B.: North-south aligned equatorial airglow depletions, Journal of Geophysical Research: Space Physics, 83(A2), 712-716, 1978.

Woodman, R. F. and La Hoz, C.: Radar observations of F region equatorial irregularities, Journal of Geophysical Research: Space Physics, 81, 5447-5466, 1976.

Yokoyama, T., Hysell, D. L., Otsuka, Y., and Yamamoto, M.: Three-dimensional simulation of the coupled Perkins and Es-layer instabilities in the nighttime midlatitude ionosphere, Journal of Geophysical Research: Space Physics, 114, A03308, doi:10.1029/2008JA013789, 2009.

405

Zabotin, N. A., and Wright, J. W.: Phase structure functions for ionospheric radio sounding: Dependence on irregularity scale, Radio Science, 39, RS2003, doi:10.1029/2003RS002882, 2004.

Zheng, H., Li, L. and Li, F.: Study about Ionospheric Effects on Spaceborne SAR Azimuth Imaging, Journal of electronics \& information technology (in Chinese), 30: 2085-2088, 2009. 
Ann. Geophys. Discuss., https://doi.org/10.5194/angeo-2019-64

Annales

Manuscript under review for journal Ann. Geophys.

Discussion started: 26 April 2019

(c) Author(s) 2019. CC BY 4.0 License.

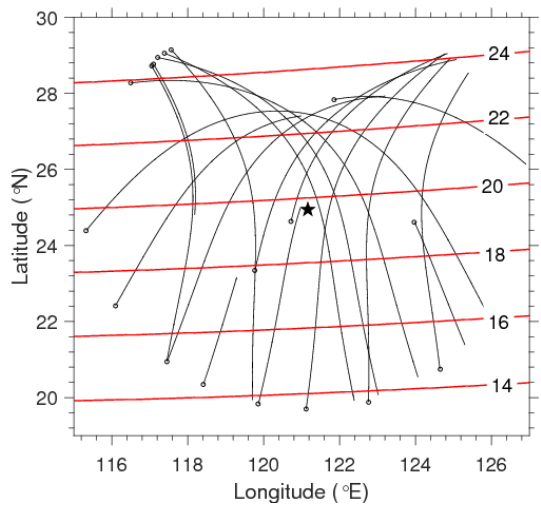

Fig. 1. IPP tracks of GPS satellites with $30^{\circ}$ elevation cutoff from Taoyuan during 18:00 06:00 local time on 20th

March 2003. The star shows the location of the GPS receiver. The dots express starting positions of the tracks.

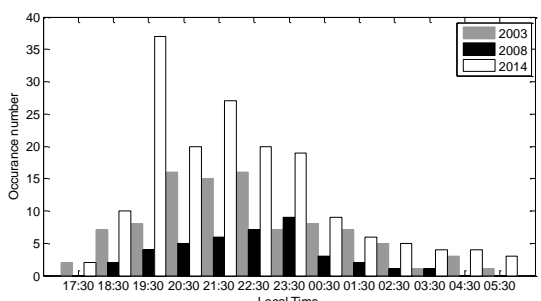

Fig. 2. Distribution of the first observed times for irregularity traverses. Most of the times fall in 19:00-24:00 LT

bin.
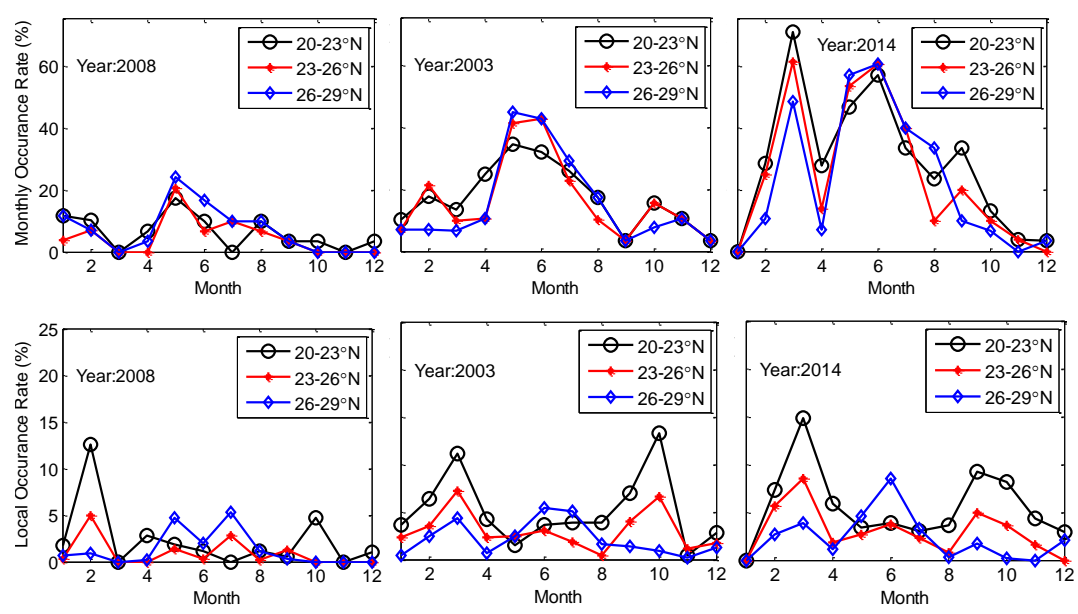

Fig. 3. Monthly and local occurrence rates of irregularities in equatorial anomaly crest. 
Ann. Geophys. Discuss., https://doi.org/10.5194/angeo-2019-64

Manuscript under review for journal Ann. Geophys.

Discussion started: 26 April 2019

(c) Author(s) 2019. CC BY 4.0 License.

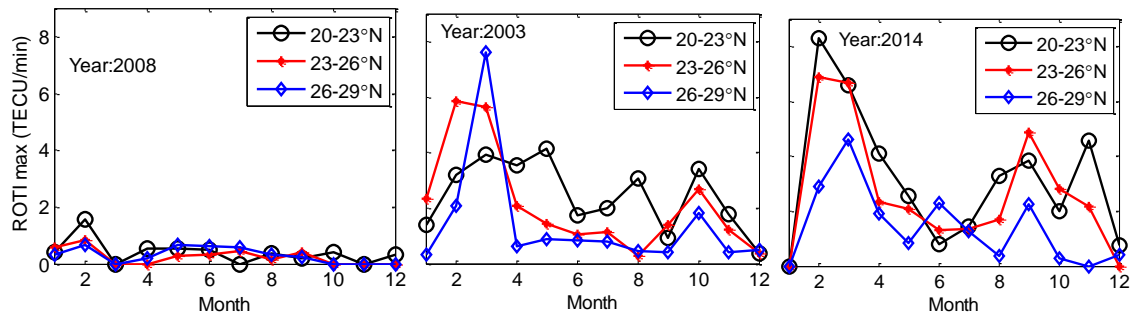

Fig. 4. Seasonal variations of ROTI maximum for irregularity traverses in equatorial anomaly crest.

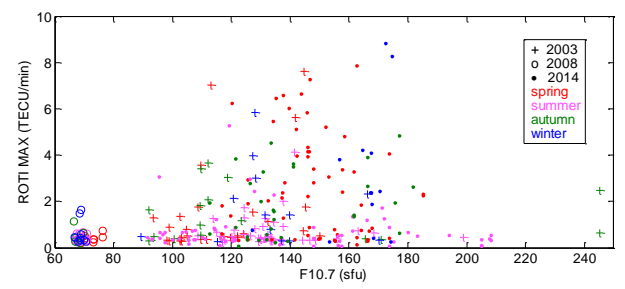

Fig. 5. Scatter plot of ROTI maximum for irregularity event versus F10.7 for different solar activity years.

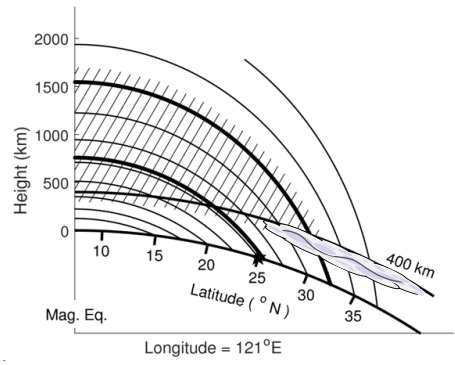

Fig. 6. A sketch of the plasma bubble and the earth's magnetic field lines at the meridian plane in Taoyuan. 